\title{
Mechanical and physical performance of cowdung-based polypropylene biocomposites
}

\begin{abstract}
In this work, potential of cow dung (CD) as a reinforcing material was evaluated. CD was blended in different ratios up to $50 \mathrm{wt} \%$ with polypropylene (PP) using Brabender twin-screw compounder. The results show a steady decline in the biocomposite tensile and impact strength with increasing CD loading. In contrast, the storage modulus ( $\left.\mathrm{E}^{\prime}\right)$, flexural modulus, and water absorption capacity of the biocomposites increased with increasing CD loading. Furthermore, it was revealed by SEM that the failure of the PP/CD biocomposites at higher filler loading was because of the week interfacial bonding. Results established that the properties of PP/CD biocomposites are a function of CD loading.
\end{abstract}

Keyword: Cowdung-based polypropylene biocomposites; Cow dung; Biocomposite 